\title{
Research on Forecasting Model of Ink Printing and Packaging Material Jia Wang
}

Yunnan Open University, Kunming Yunnan,60500

\author{
Keywords: Forecasting Model, Ink Printing, Packaging Material
}

\begin{abstract}
The previsualization of fluorescent oil half-tone prints is a key issue in the color imaging landscape. This paper aims to establish a theoretical model for predicting the color rendering of uniform fluorescent ink halftone prints with different concentrations under normal ink infiltration conditions; under the assumption that the ink is a non-scattering medium and the refractive index of the ink is approximately equal to the refractive index of the paper In this paper, the split-length Clapper-Yule spectral reflectance model of the fluorescent ink half-tone images under the penetration of ink was established by using the split-length theory that light was different in the length of the scattering and migration path between the ink and the paper.
\end{abstract}

\section{Introduction}

In anti-counterfeit printing, many fluorescent inks are printed on ordinary inks, because they can better make the fluorescent inks receive ultraviolet light, thereby producing visible fluorescence. When the first layer of ink is printed on the paper, the ink penetrates into the paper due to the printing pressure and the structure of the paper, forming an ink permeation layer between the ink and the paper. Due to the presence of the ink penetrating layer, the reflectance of the entire fluorescent halftone print also changes. This requires the establishment of a Clapper-Yule model that takes into account the underlying general ink penetration of fluorescent halftone prints. In order to simplify the established model, it is assumed that there is penetration between ordinary ink and paper, and there is no penetration between fluorescent ink and ordinary ink. Assuming a paper-ink permeation layer interface, the reflectance between the ink permeation layer and the ink interface is negligible [1].

\section{Ink penetration layer and the overall reflectance of the paper}

Based on the penetration of the ink in the blot, the ink penetration model should include the following media layers: air, ink, ink penetration, and clean paper. Assuming that the physical density of the ink is $\rho 0$, the ink penetrates from the top layer $\mathrm{z} 1$ of the paper base to the layer $\mathrm{z} 0$. The ink permeation law can be described in many different models. Let ink distribution be in the $\mathrm{z}$ direction only. See ink density in z direction as $\rho(z)$. The light absorption portion per unit length is represented by an absorption coefficient $\mathrm{K}$. The scattered light component per unit length is represented by the scattering coefficient $S$, the forward scattering coefficient is represented by $\beta$, and the backward scattering is represented by $1-\beta[2]$ :

$$
\rho(\mathrm{z})=\rho 0 \mathrm{z} 0<\mathrm{z}<\mathrm{z}
$$

The absorption and scattering coefficients of paper and ink are represented by Kp, Sp, Ki, and Si. The absorption and scattering coefficients in the ink permeation layer are

$$
\begin{gathered}
K=K p+K i, S=S p+S \\
D i=[K+(1-\beta) S] i d z-(1-\beta) S i d z
\end{gathered}
$$

\section{Propagation of layered fluorescent ink layer}

The difference between fluorescent ink and ordinary ink is that it can generate fluorescence flow, making the print more colorful. Now we analyze the light propagation of fluorescent ink prints. In order to simplify the analysis and make the process more intuitive, we need to make several assumptions. We assume that the generation of fluorescence occurs on a very thin layer in the 
middle of the ink layer, which absorbs part of the incident light and then inspires a part of the fluorescence flow. From a microscopic point of view, the fluorescence generated is in all directions. Since the ink layer is relatively thin, it is assumed that the excited fluorescence flow only has two directions, upward and downward. It is also assumed that the resulting fluorescent flow will no longer be absorbed by the ink. Let the incident light be I i0 , and the propagation in the ink layer is mainly divided into three parts [3]:

The part of the light incident on the ink surface is reflected back by I i0 rs, where rs is the internal reflectivity of the print. 2) A part of the light refracted into the ink layer is absorbed by the fluorescent molecules and converted into visible radiation. This part of the light exits the visible light intensity after passing through the fluorescent ink layer of thickness $d$ satisfying the following equation:

$$
\begin{gathered}
\mathrm{I} 1=\exp (\text { Acd)I0, I } 2=\mathrm{e} x p(\text { Fcd)I } \\
\text { I }=\mathrm{I} 1+\mathrm{I} 2=\exp (\text { Mcd)I }
\end{gathered}
$$

Among them: I1 - the intensity of the light stream absorbed by the ink layer; I2 - the intensity of the visible light stream converted from the absorbed light stream; I0 - the intensity of the incident light, c - the density of the ink like fluorescent substance, $d$ - the thickness of the ink image , $M$ Fluorescence Density Matrix, which consists of the superposition of an absorption matrix A that reflects the absorption of an ink image and a generated fluorescence matrix $F$.

$$
\mathrm{K} \mathrm{i,j}=-\varepsilon(\lambda \mathrm{j}) \mathrm{f}(\lambda) \cdot \lambda \mathrm{Q}
$$

In the formula: $\varepsilon(\lambda j)$-the extinction coefficient of the ink image; $f(\lambda i)$ - the normalized fluorescence spectrum of the fluorescent molecule; the $\lambda$-wavelength wavelength; the quantum yield rate of the $\mathrm{Q}$-fluorescent ink image.

To simplify the study, we assume that the visible light produced by the fluorescent ink has only upward and downward directions [4], and the two parts of light are equal. From the theory of physical optics, the upward or downward visible light produced by the fluorescent ink will undergo multiple scattering within the ink layer (see Figure 1). The difference between the two is that the upward optical flow first reaches the ink-air interface and then undergoes multiple intra-membrane reflections, while the downward optical flow reaches the interface formed by the ink and the paper substrate before undergoing multiple reflections. Since the reflection characteristics of the two interfaces are different, the two types of light experience different propagation paths. The specific rules are analyzed as follows: Let rg be the reflectivity of the paper-based surface, and ri be the internal reflectance of the interface between the fluorescent ink layer and air. Using the theory of multiple reflections of light in a thin film, from Figure 1, we can obtain the visible fluorescence intensity emitted upward and downward from the upper surface of the ink and the interface formed between the ink and the paper, respectively $(\mathrm{I} \leftarrow \leftarrow$, I $\leftarrow \uparrow, \mathrm{I} \uparrow \leftarrow$, I $\uparrow \uparrow$ ) expressions:

$\mathrm{I} \leftarrow \leftarrow=12$ I2(1 -ri)+ 12 I 2 ri rg(1 -ri)+ 12 I2 r2 ir 2 g(1-ri) +...=2I (2( 11--rirrig)) (4 )I $\leftarrow \uparrow=$

12 I2 ri(1 -rg)+ 12 I2 r2 irg(1 -rg)+ 12 I 2 r3 i r2 g (1 -rg)+...=I2 ri(1 -rg)2( 1-rir g) (5)I $\uparrow \leftarrow=12$

I2 rg(1 -ri)+ 12 I 2 r2 g ri(1 -ri)+ 12 I 2 r3 g r2 i (1 -I $\uparrow \uparrow=12$ I2(1 -rg $)+2$ I2 rg ri(1 -rg $)+12$ I2 r2 $\mathrm{g}$

\section{Halftone fluorescent ink print color prediction model}

Considering the penetration and expansion of the ink, the reflectance factor of the paper in the ink layer and the blank space is regarded as a function of the coverage of the ink layer of the dot [8], which is represented by $\mathrm{Ri}(\mathrm{Fi})$ and $\mathrm{Rp}(\mathrm{Fi})$, respectively. The corresponding $\mathrm{M}$ urr ay-Davies equation is:

$$
\mathrm{R}(\mathrm{Fi})=(1-\mathrm{Fi}) \mathrm{R} p(\mathrm{Fi})+\mathrm{FiR} \mathrm{i}(\mathrm{Fi})
$$

The transverse scattering law of the beam in the paper base, with an iteration approximation, there are reflectance factors at the blank paper between the dot ink layer and the dot:

$$
\begin{aligned}
& \mathrm{Ri}(\mathrm{Fi})=\operatorname{rg} \mathrm{Ti}[1-(1-\mathrm{T} \mathrm{i}) \mathrm{Fi}] \\
& \mathrm{Rp}(\mathrm{Fi})=\operatorname{rg}[1-(1-\mathrm{Ti}) \mathrm{Fi}]
\end{aligned}
$$

Find the new reflection factor of the blank paper between the ink dot and halftone dot of the 
halftone fluorescent ink print:

$\mathrm{Ri}(\mathrm{Fi})=\operatorname{rg}(1-\mathrm{rg})(1-\mathrm{rs})(1+\mathrm{ri})$ ex p $(\mathrm{F} 2(\mathrm{~cd} 1)-+\mathrm{ri}(\mathrm{r} 1 \mathrm{~g})-\mathrm{rs})(1-\mathrm{rg})[1$ - exp (Acd ]] 11-(1-rg)(1-rs)(1+ri)ex p(Fcd)+(1-rs)(1-rg)[1-exp(Acd)]2(1-) Ri rg) Fi Rp(Fi)=rg 1 - 1-(1 -rg)(1 -rs)(1+ri)e xp(Fcd)+(1-rs)(1 -rg)[ 1 -exp( Acd)]2(1 -ri rg) Fi

We have come up with a new Mürr ay-Davies color prediction model for fluorescent ink halftone prints.

\section{Murray Davies model}

The using the parameters such as ink surface reflectance, internal reflectance, paper-based reflectance, and ink density in related literature, computer simulation software Mathematica was used to simulate the established Murray-D for halftone fluorescent ink prints. The reflectivity rule reflected by av ies model is numerically analyzed [5].

The three reflectance curves are basically in line with the actual situation, especially the total reflectance curve. The Murray-Davies model requires uniform ink color, but this is not the case because of ink dot gain problems. Dot enlargement includes the physical enlargement of the dots due to the diffusion of the ink on the surface of the paper and the optical enlargement of dots due to scattering of light under practical conditions. At present, there are mainly two types of halftone reflectivity prediction models that take into account dot gains. The first is the empirical model, which is mainly based on the empirical analysis of factors (such as the Yule-Nielsen factor $\mathrm{n}$ ) or data analysis of a large number of prints. These empirical models are very useful for printers to actually correct and expand color management methods. Another model is called the mechanism model. It is mainly a matter of the physical or chemical mechanism in the printing process. This type of model mainly guides scholars and engineers in the improvement of printing devices. The establishment of this Murray-Davies reflection model based on fluorescent ink belongs to the mechanism model. In order to make the model more perfect, it needs to be combined with the empirical model to add the empirical factor. This will continue to be researched and explored in future work.

It is assumed that the fluorescence spectrum and the absorption spectrum of the fluorescence image do not overlap significantly, that is, the generated fluorescence is not reabsorbed by the fluorescent molecules. Therefore, the reflected light of the fluorescence image is divided into two independent parts, ie, the main light flow that is reduced due to absorption by the fluorescent molecules and the visible fluorescence flow that is generated due to the absorption of the main light flow, and the two parts do not interfere with each other. However, both the fluorescence flow and the main light flow are absorbed by ordinary inks, and the transmittance is t. The Clapper-Yule model of fluorescent flow is actually the Clapper-Yule model obtained by replacing the incident light with visible fluorescence. Since the fluorescence of the entire image layer is approximated by the middle c-plane, the fluorescence emitted upward and downward, as well as their propagation inside the image and paper, can be used to determine the fluorescence emitted upward. Although we are dealing with a halftone printing, we only consider fluorescence here. When fluorescence is reflected back by the paper substrate for the first time, it is expressed by the amount of 1-a+atu from the halftone print for the first time. The part of the exit. According to careful analysis, it can be found that if the light reflected back by the paper substrate is emitted from the ink image portion for the first time, only the ordinary ink image absorbs fluorescence, and the fluorescent ink image does not absorb fluorescence. Therefore, the transmittance here is Actually equal to 1 . Since the fluorescent ink is printed on the ordinary ink, the upward scattered fluorescence is not absorbed when it is first emitted, but is absorbed only in the subsequent exit.

\section{Conclusion}

Fluorescent inks are used more and more widely in the field of anti-counterfeiting printing due to their unique color rendering properties. Therefore, in order to establish an efficient color management system for fluorescent ink prints and to improve anti-counterfeiting quality, it is 
necessary to fundamentally study the coloration mechanism of fluorescent ink prints. In this paper, the famous Murray-Davies reflection model is used to establish a color prediction model for fluorescent ink prints. A predictive model for fluorescent half-tone prints considering the penetration of ink was established, which provided a theoretical basis for the quality control of printed matter, which can better improve the quality of printed images and improve quality control.

\section{Acknowledgements}

Screen Soft Proof Analysis Based on ICC Profiles, Yunnan Province Science and Technology Department Applied Basic Research Youth Project

Project Number: 2015FD039

\section{References}

[1] YANG L, K RUSE B, M IKLAVC IC S J.Revised Kub elk a-M unkTheory :II A S tudy of Ink Penetration in Ink-jet Printing [J] . 2007,(12):12-21.

[2] K UBE LKA P , M UNK F .Ein Beitrag Zu r Optik Der Farb ans triche, Zeits [J] .F Techn Physik, 2003,(12):593-601.

[3] K UBELKA P .New C ontributions to the Optics of Lintensily Lights cattering $M$ aterials.Part I [J] .Jou rn al of the Optical S ociety of America, 1998, 38(5):448-457.

[4] K UBELKA P .New C contributions to the Op tics of Linten selyLlight-scattering $M$ aterial , part II :Non-h om ogeneous Layers [J]. Journal of the Optical Society of America, 1954, 44(4):330-335.

[5] YANG L , KRUSE B.Revised Kubelka-M u nk Theory I .Theoryand Applications [J] .Journal of th e Optical Society of America ,2004, 21(9):1933-1941.Journal of th e Op tical Society of America , 2004, 21(10):1942-1952. 